The Astrophysical Journal, 999:L1-L3, 2000 January 1

(c)2000. The American Astrological Society. All rights reserved. Printed in U.S.A.

\title{
HEATING OF THE INTRACLUSTER GAS IN THE TRIANGULUM AUSTRALIS CLUSTER
}

\author{
Maxim L. Markevitch ${ }^{1}$, Craig L. Sarazin, Jimmy A. Irwin \\ Astronomy Department, University of Virginia, Charlottesville, VA 22903; mlm5y, cls7i, jai7e@virginia.edu \\ To appear in ApJ Letters, 1996 November 20
}

\begin{abstract}
$A S C A$ and ROSAT X-ray data are used to obtain two-dimensional maps of the gas temperature, pressure and specific entropy in the Triangulum Australis cluster of galaxies. We find that this hot $\left(T_{e}=10.3 \pm 0.8 \mathrm{keV}\right)$ system probably has a temperature peak $\left(T_{e}>12 \mathrm{keV}\right)$ at the cluster core, which approximately corresponds to the adiabatic relation. An underdense gas sector, found in the ROSAT cluster image eastward of the core, has a higher temperature than average at that radius. At this higher temperature, the gas pressure in this region is equal to that of the rest of the cluster at the same radius, but the specific entropy of this gas is significantly higher (although the temperature difference itself is only marginally significant). We speculate that the existence of this region of underdense high-entropy gas, as well as the adiabatic central temperature peak, indicate recent or ongoing heating of the intergalactic medium in this cluster. The most probable source of such heating is a subcluster merger, for which the hydrodynamic simulations predict a qualitatively similar temperature structure. We point out that entropy maps can provide a physically meaningful way of diagnosing merging clusters and comparing the predictions of merger simulations to the data.
\end{abstract}

Subject headings: galaxies: clusters: individual (Triangulum Australis) — intergalactic medium — Xrays: galaxies

\section{INTRODUCTION}

Spatially resolved measurements of the gas temperature in clusters of galaxies can provide valuable information on the dynamical history of these systems, pointing to those clusters with recent or ongoing merger activity (see, for 'example, hydrodynamic merger simulations by Schindler '\& Müller 1993; Roettiger, Burns, \& Loken 1993; Evrard, Metzler, \& Navarro 1996). Such measurements for hot 'clusters have become possible with the advent of $A S C A$ with its combination of angular and spectral resolution and the wide energy band of $0.5-10 \mathrm{keV}$ (Tanaka, Inoue, '\& Holt 1994). Two-dimensional gas temperature maps of several clusters obtained with $A S C A$ using independent techniques were presented, e.g., in Arnaud et al. (1994), 'Markevitch et al. (1994), Markevitch (1996), Churazov et al. (1996), Honda et al. (1996), Markevitch \& Vikhlinin (1996), and Henriksen \& Markevitch (1996). The latter authors detected a prominent asymmetric temperature pattern in the cluster A754, confirming with improved accuracy a similar ROSAT finding of Henry \& Briel (1995). The temperature nonuniformity in A754, together with its complex X-ray image, indicated that it is undergoing a major merger. A temperature structure of this kind was also found in A3558, as well as in Coma. On the other hand, no significant asymmetric features were detected in other well-resolved clusters, A2256, A2319 and AWM7, except for the lower temperatures at the positions of the infalling subunits in the former two systems. A significant radial temperature decline at large radii was found with $A S C A$ in several clusters; for some of them, most notably A2256, ROSAT PSPC provided an independent confirma- tion (refs. above).

Here we report on the detection of a possible merger signature in another cluster, Triangulum Australis. It is a relatively nearby $(z=0.051)$ bright, hot system, which was overlooked in the optical band due to its low Galactic latitude. It was first discovered as an X-ray source (McHardy et al. 1981). From an EXOSAT observation, Edge \& Stewart (1991) obtained an average temperature of $8.0_{-1.3}^{+1.4}$ $\mathrm{keV}$ and an absorption column of $2.5_{-1.0}^{+1.8} \times 10^{21} \mathrm{~cm}^{-2}$, somewhat higher than the Galactic value of $1.25 \times 10^{21}$ $\mathrm{cm}^{-2}$ (Heiles \& Cleary 1979). The cluster has since been observed with ROSAT, which produced a high-quality image and an accurate measurement of the absorption column, and with $A S C A$, which provided a gas temperature map. These results are presented below. At the cluster's redshift, $1 h^{-1} \mathrm{Mpc}=25^{\prime} .4$, using $H_{0} \equiv 100 h$ $\mathrm{km} \mathrm{s}^{-1} \mathrm{Mpc}^{-1}$.

\section{ROSAT RESULTS}

The cluster was observed with ROSAT PSPC for $7 \mathrm{ks}$ in September 1992. We have used the method and code of Snowden et al. (1994) to obtain the flat-fielded images of the source in the energy bands R2-R7 (0.20-0.42-0.520.70-0.91-1.32-2.01 keV), free of non-cosmic background. The cosmic component of the background was then calculated individually in each energy interval using the outer parts of these images, with the assumed accuracy of $5 \%$ (Markevitch \& Vikhlinin 1996). Fluxes from these images (excluding the poorly calibrated band R3) were fitted to estimate the cluster temperature and the absorption col-

\footnotetext{
${ }^{1}$ Also IKI, Profsoyuznaya 84/32, Moscow 117810, Russia

${ }^{2}$ Quoted errors are $90 \%$ one-parameter intervals throughout the paper.
} 
umn. We did not include estimates of the PSPC calibration uncertainty to our analysis, since our primary interest in the ROSAT data is to obtain the image and the absorption column value, well-determined by PSPC. Our best-fit $N_{H}$ value is $1.42_{-0.25}^{+0.21} \times 10^{21} \mathrm{~cm}^{-2}$, and $T_{e}=7$ (4-17) $\mathrm{keV}$, the large temperature error being due to the high $N_{H}$. The absorption column is consistent with the Galactic value and is marginally allowed by EXOSAT. We also attempted to constrain the spectrum in the inner $r=1^{\prime} .5$ circle (half the core radius) of the cluster, to see if there is any trace of a cooling flow to which PSPC is highly sensitive. We approximately corrected for the energy dependence of the PSPC PSF (Hasinger et al. 1992), which for this small radius makes a noticeable difference in the spectral fit. Fixing $N_{H}$ at its value for the whole cluster and using only $0.5-2 \mathrm{keV}$ data, we obtain for this region $T_{e}=8.2(4.2-\infty) \mathrm{keV}$. Freeing $N_{H}$ and including lower energies, the temperature is $T_{e}=5.0(2.4-\infty) \mathrm{keV}$ and $N_{H}=1.7 \pm 0.5 \times 10^{21} \mathrm{~cm}^{-2}$. Inclusion of a cooling flow component does not improve the fit significantly; the best-fit inflow rate is $7(0-33) h^{-2} M_{\odot} \mathrm{yr}^{-1}$ with a similar best-fit absorption. The cluster image does not have a brightness peak characteristic of a strong cooling flow, and our $A S C A$ spectra (below) do not require a cooling flow component either. We conclude that there is no indication of a significant cooling flow in the center.

The cluster image in the $0.5-2 \mathrm{keV}$ band is presented by contours in Fig. 1. Its X-ray brightness peak has coordinates $\alpha=16^{\mathrm{h}} 38^{\mathrm{m}} 20^{\mathrm{s}}, \delta=-64^{\circ} 21^{\prime} 22^{\prime \prime}(\mathrm{J} 2000)$. Assuming $T_{e}=10 \mathrm{keV}$ and an iron abundance of 0.26 (average $A S C A$ values, see below) and the absorption column derived above, the $0.5-2 \mathrm{keV}$ cluster luminosity within $r=1 h^{-1} \mathrm{Mpc}$ is $2.1 \pm 0.1 \times 10^{44} h^{-2} \mathrm{erg} \mathrm{s}^{-1}$. Fitting the brightness profile in the range of radii $0-25^{\prime}$ by a $\beta$-model of the form $I_{x}(r) \propto\left(1+r^{2} / a_{x}^{2}\right)^{-3 \beta+1 / 2}$ (Jones \& Forman 1984) and ignoring the asymmetry of the image, we obtained $a_{x}=3^{\prime} .5 \pm 0^{\prime} .2\left(0.143 h^{-1} \mathrm{Mpc}\right)$ and $\beta=0.63 \pm 0.02$. We will use the derived $N_{H}$ value and the ROSAT image in our $A S C A$ analysis below. Note the apparent underdense sector east of the cluster center, which will be discussed below. At the off-center distances of $3-10^{\prime}$, the surface brightness in this sector is about 2 times lower than that at the same radius on the opposite side of the cluster.

\section{ASCA ANALYSIS}

ASCA observed Triangulum Australis in March 1995 in two pointings with $11.3 \mathrm{ks}$ and $6.7 \mathrm{ks}$ GIS useful time, which were offset by $7^{\prime}$ from one another. The SIS has low sensitivity at high energies relevant for this hot cluster, and was operated in 1-CCD mode which covers only $11^{\prime} \times 11^{\prime}$ area, so we limited most of our analysis to the GIS data. Using the energy band $1-11 \mathrm{keV}$, fixing the absorption column at the ROSAT best-fit value and fitting both pointings simultaneously, we obtained an average cluster temperature of $10.3 \pm 0.8 \mathrm{keV}$ and an iron abundance of $0.26 \pm 0.10$ (relative to Allen 1973). The EXOSAT temperature value $\left(8.0_{-1.3}^{+1.4} \mathrm{keV}\right)$ is somewhat lower; however, assuming their higher best-fit value of $N_{H}$, our temperature becomes $8.9 \mathrm{keV}$, consistent with EXOSAT.

For the reconstruction of the two-dimensional map of the cluster gas temperature, the method described in de- tail in Markevitch (1996, and references therein) has been used. It consists of simultaneous fitting of the temperature in all image regions, using a $0.5-2 \mathrm{keV}$ ROSAT PSPC image as a brightness template and modeling the $A S C A$ mirror scattering. We restricted our spatially resolved analysis to the $2.5-11 \mathrm{keV}$ energy band due to the poor PSF calibration at lower energies. (A temperature value for the whole cluster obtained from this energy band is $10.8_{-1.1}^{+1.3}$ $\mathrm{keV}$.) The estimated systematic uncertainties of the background normalization $(20 \%, 1 \sigma)$, the PSF model's wings (15\%) and core $(5 \%)$ and the ASCA effective area (5\%) were taken into account, as well as the uncertainty due to the relative displacement of the $A S C A$ and $R O S A T$ images and the statistical error of the ROSAT image. The nearest bright X-ray source to Triangulum Australis is $3^{\circ}$ away, so the possibility of stray light contamination is excluded (Ishisaki 1996).

The image was divided into 3 concentric annuli with $r=0-3-12-23^{\prime}$ centered on the brightness peak. The second and third annuli were divided into 5 and 4 sectors, respectively, with one of the sectors coinciding with the low-brightness region. The spectra from each region, each pointing and each detector were binned in 7-10 energy intervals for adequate Gaussian statistics. A uniform temperature over each image region was assumed. We obtained $\chi_{\min }^{2}=149$ for 224 d.o.f. for the simultaneous fit. The low reduced $\chi^{2}$ indicates that our conservative compound errors are, if anything, slightly overestimated. The confidence intervals were then estimated by Monte-Carlo simulations, including systematic errors. Figures 1 and $2 a$ show the resulting temperature map. Two sectors to the north and west of the outer ring which were poorly covered by $A S C A$ pointings are not shown, but they were accounted for in the fit for completeness. There are two features in the map which will be discussed below - a significant temperature peak in the cluster core, and a higher temperature in the cluster low-brightness sector 2 compared to other regions at this radius. (The contributions to the total $\chi_{\min }^{2}$ due to these regions are 24 and 14 for 28 data bins, respectively.) The latter temperature difference by itself is statistically insignificant; however, due to the coincidence with the depression of the gas density, the region's specific entropy (Fig. $2 c$ ) is significantly different from the average at this radius. The entropy values in sectors 2-6 could not be drawn from the same gaussian distribution at the $95 \%$ confidence, due to the single large deviation in region 2 .

We note that the $A S C A$ data alone do not require as large a central temperature peak. As a consistency check, we performed a fit with the emission measure of the central region (relative to other regions) being an additional free parameter. Normally, the $0.5-2 \mathrm{keV}$ relative brightness in different regions is predetermined by the ROSAT image in our technique. (Note that the ROSAT-ASCA relative normalization is not used.) The resulting best-fit relative brightness in the central annulus was $22 \%$ higher than that from ROSAT, and the central temperature was lower, with the temperatures in all other regions, as well as error bars, increasing (dotted symbols in Fig. 1a). However, both quantities were consistent within their 90-95\% confidence intervals with the temperature obtained above and the ROSAT brightness, respectively. Larger temper- 
ature errors in such a fit are natural because the important piece of information provided by the ROSAT image is withdrawn. We have checked the possibility that ROSAT underestimates the relative emission measure in the $r<3^{\prime}$ region by $20 \%$, for example, due to a greater absorption toward the center (a factor of $>2$ increase is needed). The ROSAT best-fit $N_{H}$ in the central $r=3^{\prime}$ region is $1.55_{-0.39}^{+0.35} \times 10^{21} \mathrm{~cm}^{-2}$, far short of what is needed to produce such effect. The PSPC finite angular resolution depletes the flux in the image peak only by $\sim 2 \%$, which is absorbed by the assumed $A S C A$ PSF uncertainty. A cooling flow, which would make the ROSAT image inadequate for our purpose, is not required by ROSAT (above), nor does it make any improvement to either the GIS or the SIS fit. (The short SIS exposure covering the central region does not provide useful constraints for the main hot component.)

An optical image of Triangulum Australis shows that there is a $\mathrm{cD}$ galaxy $0^{\prime} .3$ from the $\mathrm{X}$-ray brightness peak. If it has an active nucleus, it may add a nonthermal component to the central flux. However, a $4.85 \mathrm{GHz}$ radio image (Condon et al. 1993) shows no source at the galaxy's position with an upper limit of about $30 \mathrm{mJy}$ (two orders of magnitude below the luminosity of the $\mathrm{cD}$ galaxy NGC 1275 in the Perseus cluster). Therefore, a significant nonthermal flux from this galaxy is unlikely, unless it is a radio-quiet AGN, unusual in a cluster center. The best-fit normalization of an additional X-ray component with a typical power-law AGN spectrum is zero, although anything more exotic (for example, a heavily self-absorbed AGN with $\left.L_{x}(2-10 \mathrm{keV}) \sim 2 \times 10^{43} h^{-2} \mathrm{erg} \mathrm{s}^{-1}\right)$ cannot be excluded, due to the $A S C A$ 's limited energy band.

We also tried to fit the relative brightness in sector 2 as another free parameter, which practically did not change the temperature and the normalization in this region compared to the value fixed by ROSAT. Given all the above, we will assume that the central discrepancy is probably a statistical deviation, and our temperature measurement using the ROSAT image is correct, although it appears to be model-dependent. Our major conclusions will hold even without the central temperature peak.

\section{DISCUSSION}

We will now discuss the implications of our temperature measurement. Interestingly, from the ROSAT image alone, which is rather circular except for the lowbrightness sector, one might expect that this sector has a higher temperature, if the gas is in pressure equilibrium and the gravitational potential of the cluster is reasonably smooth and symmetric. Pressure variations not supported by gravity would disperse in roughly their sound crossing time $\left(\sim 3 \times 10^{8} h^{-1}\right.$ yr for the low brightness sector). Since this sector presumably contains lower density gas, it must have a higher temperature to maintain a pressure similar to the adjoining sectors. As our temperature map indicates, the gas in this region is indeed likely to be hotter. It is useful to compare the gas pressure and specific entropy in different regions, which is done in Figure $2(b, c)$. Here, the entropy per particle is defined as $\Delta s \equiv s-s_{0}=\frac{3}{2} k \ln \left[\left(T / T_{0}\right)\left(\rho / \rho_{0}\right)^{-2 / 3}\right]$, the subscript
0 denoting central values. The emission measure-weighted average density in each sector was crudely estimated as the value from the symmetric $\beta$-model distribution multiplied by the ratio of the square root of the actual X-ray brightness to that in the model. Figure $2 b$ indicates that the gas pressure is nearly constant at a given radius. Thus, the gas could be nearly in hydrostatic equilibrium with a symmetric gravitational potential. Note, however, that the gas in region 2 is less dense than the average in the middle annulus and would be expected to rise buoyantly on a timescale of $\sim 10^{9} h^{-1}$ yr. Thus, the surface brightness distribution in Figure 1 is not consistent with a stable equilibrium distribution, and is likely to have resulted from a transient hydrodynamic process. We note that the image can perhaps be explained as well by a very irregular underlying mass configuration, but that would certainly mean that the cluster is merging.

A given gravitational potential may support different gas configurations, which are hydrostatic but at the same time differ by their histories and their entropy distributions. The specific entropy $\Delta s$, shown in Fig. $2 c$ for our data, is a useful diagnostic of shock heating or of any other nonadiabatic process. It may provide information on how the particular, observed gas distribution has emerged. For comparison, Figure $2 c$ also shows the entropy for an isothermal $\beta$-model (arbitrarily choosing a temperature of $8 \mathrm{keV}$ of the three cooler "reference" sectors of the second annulus 3 ). This is the kind of distribution cluster gas is expected to approach if left to itself, particularly if thermal conduction is effective. Two gas regions significantly deviate from this isothermal equilibrium model: the cluster core (if we accept that our central temperature is correct) and sector 2. Another notable detail is that the gas in the core has nearly the same specific entropy as the average in the surrounding annulus. One explanation of this adiabatic temperature peak is that the core gas has recently mixed turbulently with the surrounding gas. The higher temperature in the core would then result from the adiabatic compression required to bring the pressure in the core into hydrostatic equilibrium in a given gravitational potential. The second annulus and the outer sectors have similar temperatures and different entropies, which suggests that any such mixing was confined to the central region. This is not unreasonable since the core has only about $10 \%$ of the gas within the outer boundary of our second annulus.

However, the entropy in region 2 (the low brightness sector) is higher than the average for that annulus or that of the cluster core. This could not be due to mixing; it suggests that the gas in this region underwent a recent heating event. For example, this might have been due to the passage of a shock from a subcluster merger (e.g., Schindler \& Müller 1993; Pearce, Thomas, \& Couchman 1994), or this gas may have been expelled from the core where the heating occurred. Presumably, this gas then expanded adiabatically into pressure equilibrium with the surrounding gas. Note that because the pressure has already equalized but the entropy in this region is still high, the event should have happened about $10^{9} h^{-1}$ yr ago. The same event may have heated the central gas sufficiently to produce a radially decreasing entropy gradient,

${ }^{3}$ We also chose the observed values of $a_{x}$ and $\beta$ for clarity, although they would be different for a given binding mass profile. 
after which convective mixing would act to eliminate this gradient, producing a distribution with similar specific entropy in the core and the surrounding gas. One can estimate the amount of energy which should have been injected into the gas residing in the core and region 2 , assuming that the cluster was initially isothermal at $8 \mathrm{keV}$, as $Q \approx T \Delta S \sim(3-6) \times 10^{61} h^{-5 / 2}$ erg (approximately equally distributed between the core and sector 2 ). To estimate the heating which might occur in a merger, we consider the kinetic energy acquired by the gas in two subclusters each with a total mass of $6 \times 10^{14} M_{\odot}$ infalling from a distance of 5 to $1 \mathrm{Mpc}$; this energy is about 10 times greater than required. Therefore, a merger of subclusters is a reasonable source of such heating. Hydrodynamic simulations predict that a head-on merger of subclusters should produce a hot core and an asymmetric temperature structure not unlike the observed one.

Other sources of heating might include supernova-driven galactic winds (e.g., Evrard, Metzler, \& Navarro 1996). However, the present day supernova rate expected for the stellar population in the core of this cluster is much too small to produce enough heating on the timescale limited by the possible lifetime of the observed temperature structure.

\section{SUMMARY}

Using $A S C A$ and $R O S A T$ data, we have reconstructed two-dimensional distributions of the gas temperature, pressure and entropy in the Triangulum Australis cluster. We detect a significant temperature peak in the cluster core (although its existence is somewhat modeldependent), and a likely temperature increase in the sector coincident with the underdense region of the gas found in the cluster X-ray image. The underdense region is in pressure equilibrium with the gas at the same radius but has a higher specific entropy. We interpret the obtained entropy distribution as evidence of the ongoing or recent heating of the intracluster medium, perhaps by shocks from a subcluster merger. A temperature map with higher angular resolution to look into the structure of the cluster core, as well as covering the outermost cluster regions, would be useful for understanding the processes responsible for heating of the gas in this object, and perhaps in clusters in general. The entropy distribution is a useful diagnostic of shock heating or of any other nonadiabatic process. Therefore, it would be very helpful if simulations of cluster hydrodynamics were analyzed to produce the gas pressure and entropy maps, to enable a quantitative and physically meaningful comparison to the data.

We thank the referee, A. C. Edge, for useful criticism. We gratefully acknowledge the support from NASA grants NAG5-2526 and NAG5-1891.

\section{REFERENCES}

Allen, C. W. 1973, Astrophysical Quantities (London: Athlone)

Arnaud, K. A., et al. 1994, ApJ, 436, L67

Churazov, E., Gilfanov, M., Forman, W., \& Jones, C. 1996, in X-ray Imaging and Spectroscopy of Cosmic Plasmas, ed. F. Makino, in press

Condon, J. J., Griffith, M. R., \& Wright, A. E. 1993, AJ, 106, 1095

Edge, A. C., \& Stewart, G. C. 1991, MNRAS, 252, 414

Evrard, A. E., Metzler, C. A., \& Navarro, J. F. 1996, ApJ in press

Hasinger, G., Turner, J., George, I., \& Boese, G. 1992, ROSAT Calibration Memo CAL/ROS/92-001

Heiles, C., \& Cleary, M. N. 1979, Australian J. Phys. Astroph. Suppl., 49, 1

Henriksen, M. \& Markevitch, M. 1996, ApJ, 466, L79

Henry, J. P., \& Briel, U. G. 1995, ApJ, 443, L9

Honda, H., Hirayama, M., Watanabe, M., Yamashita, K., Ohashi, T. 1996, in X-ray Imaging and Spectroscopy of Cosmic Plasmas, ed. F. Makino, in press

Ishisaki, Y. 1996, PhD thesis, University of Tokyo

Jones, C., \& Forman, W. 1984, ApJ, 276, 38

Markevitch, M. 1996, ApJ, 465, L1

Markevitch, M., \& Vikhlinin, A. 1996, ApJ, in press

Markevitch, M., Yamashita, K., Furuzawa, A., \& Tawara, Y. 1994 ApJ, 436, L71

McHardy, I. M., Lawrence, A., Pye, J. P., \& Pounds, K. A. 1981 MNRAS, 197, 893

Pearce, F. R., Thomas, P. A., \& Couchman, H. M. P. 1994, MNRAS, 268, 953

Roettiger, K., Burns, J., \& Loken, C. 1993, ApJ, 407, L53

Schindler, S., \& Müller, E. 1993, A\&A, 272, 137

Snowden, S. L. McCammon, D., Burrows, D. N., \& Mendenhall, J. A. 1994, ApJ, 424, 714

Tanaka, Y., Inoue, H., \& Holt, S. S. 1994, PASJ 46, L37 


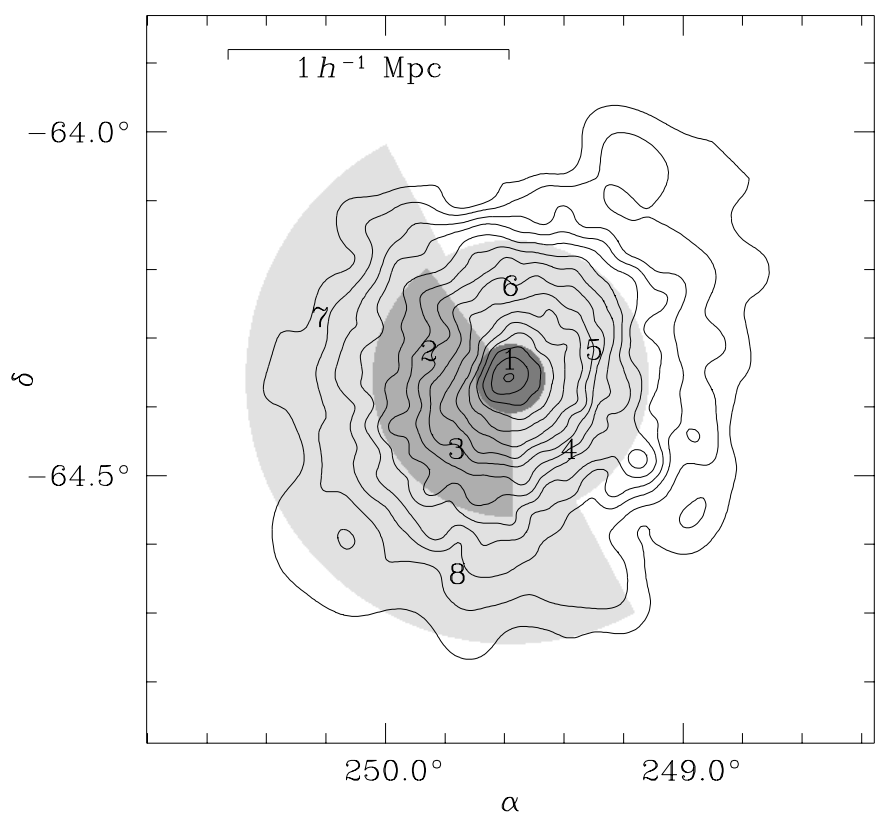

Fig. 1.- Temperature map of Triangulum Australis. Contours show the ROSAT PSPC surface brightness in the 0.5-2 $\mathrm{keV}$ band (smoothed with a Gaussian of variable width for clarity). The contours are logarithmically spaced with a factor of $\sqrt{2}$. The grayscale shows $A S C A$ temperatures (darker is hotter). Regions (whole inner circle and two outer annuli divided into 5 and 4 sectors, respectively) are numbered and their temperatures are shown in Fig. $2 a$. The two sectors of the outer annulus which were poorly covered by $A S C A$ are not shown.
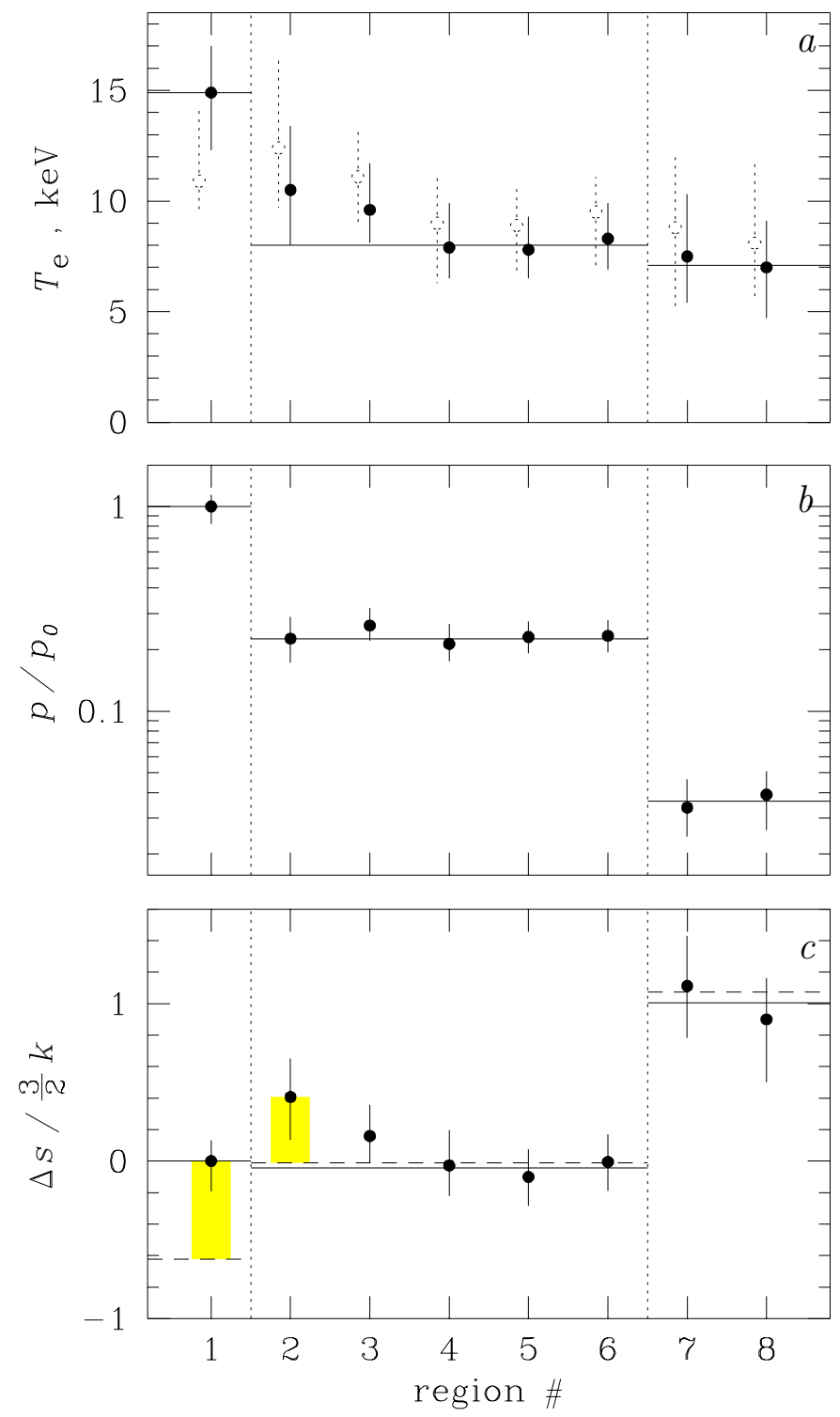

FiG. 2.- Temperature (a), gas pressure $(b)$ and specific entropy relative to the central value $(c)$ in the cluster regions numbered in Fig. 1. Errors are 90\%. The dotted symbols in panel $(a)$ correspond to a fit with the relative central brightness being a free parameter (see the text). The solid horizontal lines correspond to the average values over each annulus, excluding sectors 2 and 3 for the second annulus. The dashed line in panel $(c)$ corresponds to a symmetric isothermal $\beta$-model with $T_{e}=8 \mathrm{keV}$ (the average temperature in our "reference" sectors 4,5 and 6). The values of $\Delta s$ used for an estimate of the amount of energy injected into the gas (see text) are shown by shaded bars. 